\title{
Comparative study of airway assessment tests to predict difficult laryngoscopy \& intubation
}

\author{
Anjali Bhure $^{1}$, Ankush Wasnik ${ }^{2 *}$, Priyanka P. Deshmukh ${ }^{3}$, Yasha Tiwari ${ }^{4}$ \\ ${ }^{1}$ Professor and HOD, ${ }^{2-4}$ Junior Resident, Dept. of Anaesthesiology, NKP Salve Institute of Medical Sciences, Nagpur, Maharashtra, India
}

\author{
*Corresponding Author: Ankush Wasnik
}

Email: psychogotu@gmail.com

Received: $20^{\text {th }}$ December, 2018

Accepted: $26^{\text {th }}$ March, 2019

\begin{abstract}
Introduction: Prediction of potentially difficult airway management during pre-operative period is determined by anatomy of oropharynx \& range of movement of neck, clinically evaluated by various tests like Modified Mallampati Test, Upper Lip BiteTest, Thyromental Distance, ratio of Height to thyromental distance etc. We performed a study to compare significant direct relationship between MMT, ULBT, TMD, RHTMD \& Cormack-Lehane (C-L) scale.

Aims and Objective: 1) To elucidate the role of MMT, ULBT, TMD and RHTMD as simple bedside airway predictive tests \& to study their direct correlation with difficult laryngoscopic view, using C-L grading.

2) To compare their ability to predict difficult laryngoscopy in various combinations.

Materials and Methods: Type of study - prospective, observational \& single blind.

Sample Size: 450, 20-60 years, male/female, ASA I/II.

Pre-operatively anaesthesiologist not involved in intubation evaluated \& assessed ULBT, MMT \& TMD in sitting position. Patient induced \& laryngoscopy performed with Macintosh blade No.3 in sniffing position \& laryngoscopic view determined by C-L grading.

Result: On comparing CL-grading with other tests such as MMT, ULBT, TMD \& RH MD in assessing difficult intubation, it was observed that there was statistically significant association with $\mathrm{p}<0.05$. Kappa coefficient was highest for MMT \& RHTMD with CL-grading as compared to other tests. MMT had highest sensitivity \& lowest positive predictive value, ULBT had highest specificity \& lowest negative predictive value. MMT \& RHTMD had highest diagnostic accuracy followed by other tests.

Conclusion: We conclude that no single airway predictor test is sufficient for predicting difficult intubation. So, a combination of two or more airway predictor tests may predict difficult airway better.
\end{abstract}

Keywords: Difficult intubation, Mallampati test, Upper lip bite test, Thyromental distance, RHTMD.

\section{Introduction}

In airway management tracheal intubation using direct laryngoscopy remains Gold standard. Of all anaesthetic deaths, 30-40\% are attributed to inability to manage difficult airway. Prediction of potentially difficult airway management duringpre-operative period is determined by anatomy \& range of movement of oropharynx \& neck which is clinically evaluated by various tests like MMT, ULBT, TMD, RHTMD etc.

Modified Mallampati Test indicates significant correlation between the ability to visualise pharyngeal structures \& ease of laryngoscopy \& intubation. Its classification is based on observation of pharyngeal structures with mouth fully open \& tongue maximally protruded without phonation.

Upper Lip Bite Tests is based on fact that range \& freedom of mandibular movement \& architecture of the teeth have pivotal role in facilitating laryngoscopic intubation. It is classified according to the ability to bite the upper lip with lower teeth.

Thyromental distance \& Ratio of Height to Thyromental distance are other predictive tests for difficult laryngoscopy.

To the best of our knowledge there was no study to clarify which method predicted difficult laryngoscopy more accurately.
So, we have performed prospective, single blind study to compare significant direct relationship between MMT, ULBT, TMD, RHTMD \& Cormack-Lehane scale.

\section{Materials and Methods}

Type of Study

Prospective, observational, single-blind study.

Hospital Setting

Our hospital where this study was conducted is a tertiary care teaching institute from central India. Department of anesthesia was the primary site for this study.

\section{Duration of Study}

Study commenced in December 2015 and was completed in November 2017.

\section{Ethics Committee Permission}

The study was initiated only after obtaining permission from the Institutional Ethics Committee. (IEC)

\section{Sample Size}

450 patients of either sex (accuracy with 95\% confidence interval)

\section{Sample Size Estimation}

The reported incidence of difficult intubation ranges from 0.5 to $18 \%$. Also, the sensitivity, specificity for different tests like MMT, TMD, RHTMD and ULBT have been reported in study carried out by Shah PJ et al. (2013). These data were used to estimate the sample size for the proposed study. 
The formula used for estimation of sample size was: $\mathrm{n}=\mathrm{Z} 2 \mathrm{P}(1-\mathrm{P}) 2$

Where $Z$ is 1.96 for $5 \%$ significance level, $P$ is the targeted sensitivity or specificity, and $n$ is the $(T P+F N)$ if $P$ is sensitivity and $(F P+T N)$ if $P$ is specificity.

In the present case, sensitivity of different methods as reported in the study has been used to estimate the sample size for the study.

Accordingly, the total sample size $N$ is given by:

$N=(T P+F N) /$ Incidence rate

The sensitivity of MMT was reported as $70.15 \%$, while specificity was $61.02 \%$. Considering the incidence rate of $18 \%$ and the desired precision level of $10 \%$, the estimated sample size was 451 .

Using data for TMD (sensitivity of $7.46 \%$ and specificity of $98.06 \%$ ), and the same incidence and the precision levels, the estimate of sample size was 145 .

For RHTMD, the sensitivity and specificity were $71.6 \%$ and $92.01 \%$ respectively. Accordingly, the sample size estimate was 445.

The sensitivity and specificity of ULB were $74.6 \%$ and $91.5 \%$ respectively. Using these parameters the sample size estimate was 412.

Considering these results, a sample size of 450 should be adequate and would provide a diagnostic efficiency of tests.

Written informed consent was taken from all patients

\section{Inclusion Criteria:}

1. ASA Grade I OR II

2. Age: $20-60 \mathrm{yrs}$.

3. Elective surgeries under General Anaesthesia with intubation

\section{Exclusion Criteria:}

1. Edentulous patient.

2. Patient unable to open the mouth.

3. Pharyngolaryngeal pathology.

4. History of Thyroid/Neck surgery.

5. Limitations of Temporomandibular or Atlanto axial joint movements

6. Pregnant patient.

7. Patient refusal.

\section{Materials and Methods}

Preoperatively Anaesthesiologist not involved in intubation had evaluated and assessed the ULBT class and MMT class.

Determination of ULBT class: Patient made in sitting position. Patient was asked to bite the upper lip with the lower incisors.
Class I: Lower incisors can bite upper lip above the vermilion line.

Class II: Lower incisors can bite upper lip below the vermilion line.

Class III: Lower incisors can't bite upper lip.

ULBT of class III, was considered as markers of a potentially difficult intubation.

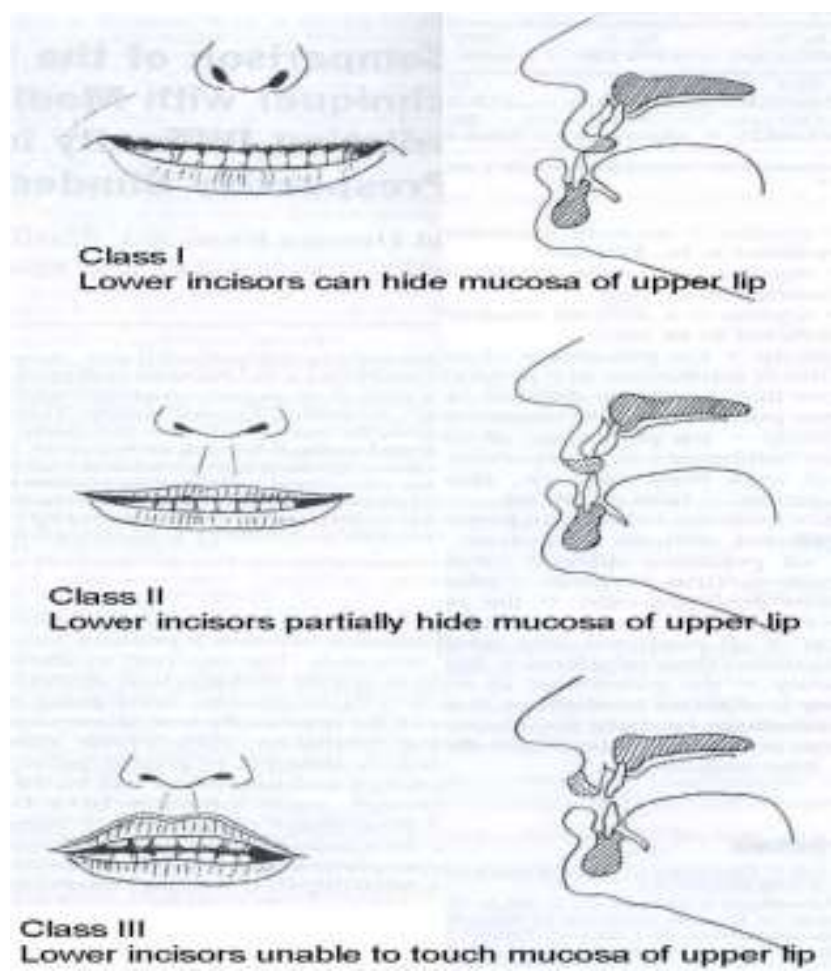

Fig. 1: Schematic frontal and lateral view of the upper lip bite test

Classification of oropharyngeal view was done according to MMT, wherein the patient was made to be in sitting position with mouth fully open and tongue maximally protruded, and patient was asked not to phonate.

Class I - Soft palate, fauces, uvula, and pillars are seen

Class II - Soft palate, fauces, and uvula are seen.

Class III - Soft palate and base of uvula

Class IV - Soft palate not visible. MMT Class III and IV was considered as markers of a potentially difficult intubation.

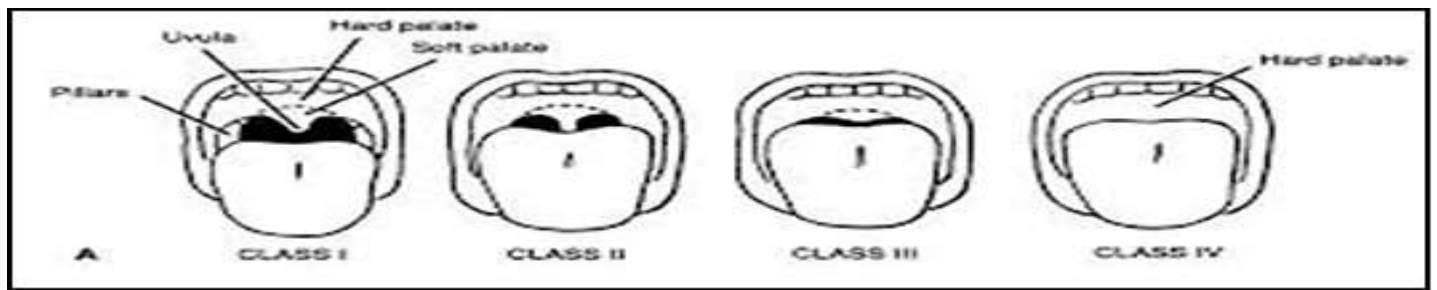

Fig. 2: Mallampatti test 
Thyromental Distance (TMD) was measured from the bony point of the mentum while the head was fully extended and the mouth closed, using a rigid ruler. The distance was rounded to nearest $0.5 \mathrm{~cm}$ and graded ${ }^{1}$ (Table A).

Class I - >6.5 cm

Class II - 6-6.5 cm

Class III- $<6 \mathrm{~cm}$

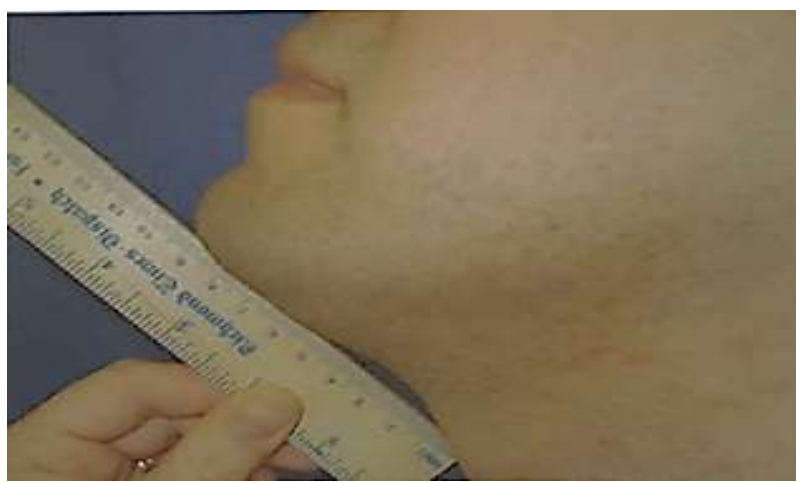

Fig. 3: Thyromental distance. Ruler measurement from thyroid cartilage to menton of mandible

Height, body weight, and body mass index (BMI) was assessed also. Height of the patient was measured in centimeters from vertex to heel with the patient standing and was rounded to the nearest $1 \mathrm{~cm}$.

Ratio of Height to Thyromental Distance (RHTMD) was calculated and graded ${ }^{2}$ (Table A) RHTMD $=$ Height (in $\mathrm{cms}$ )/TMD (in cms).

Preparation of patients included over-night fasting for 8-10 hours. On the day of surgery, in the pre-operative room written and informed consent was checked and NBM status was confirmed. Intravenous line was secured. In the OT, three lead ECG, pulse oximetry (heart rate and $\mathrm{SpO}_{2}$ ), noninvasive arterial pressure (NIBP) were connected to the patient. Patient was pre-medicated with injection Ranitidine $50 \mathrm{mg}$ iv in drip and injection Ondansetron $4 \mathrm{mg}$ iv and intravenous fluid was started.

Premedication with injection Midazolam $0.5 \mathrm{mg} / \mathrm{kg}$, injection Glyccopyrolate $0.004 \mathrm{mg} / \mathrm{kg}$, injection Fentanyl $1 \mu \mathrm{g} / \mathrm{kg}$. Induction with injection Propofol $2 \mathrm{mg} / \mathrm{kg}$ and injection Succinylcholine $2 \mathrm{mg} / \mathrm{kg}$, laryngoscopy was done with Macintosh blade no 3, with neck flexion $\left(35^{\circ}\right) \&$ head extension $\left(15^{\circ}\right)$ in sniffing position \& the laryngoscopic view would be determined using Cormack-Lehane grading system as follows:

Grade I: Full view of glottis.

Grade II: Glottis partly exposed, ant. commissure not seen.

Grade III: Only epiglottis seen.

Grade IV: Epiglottis not seen.

No external laryngeal pressure was applied while reporting the laryngeal view. C-L grades I \& II was considered as "easy intubations" grades III \& IV as "difficult intubations". Data was analysed using kappa agreement \& calculation of sensitivity, specificity, positive predictive value, negative predictive value, accuracy with their $95 \%$ confidence interval.

Table A: Grading of various predictive tests

\begin{tabular}{|l|c|c|}
\hline & Easy & Difficult \\
\hline Test & I / II & III / IV \\
\hline MMT (Mallampati test) & I / II & III \\
\hline ULBT (upper lip bite test) & I II & III \\
\hline TMD (thyromental distance) & $<23.5$ & $>23.5$ \\
\hline $\begin{array}{l}\text { RHTMD (ratio of height to } \\
\text { thyromental distance) }\end{array}$ & I / II & III / IV \\
\hline $\begin{array}{l}\text { CL GRADING (Cormack-Lehane } \\
\text { grading) }\end{array}$ & & \\
\hline
\end{tabular}

\section{Results}

Table 1: Descriptive statistics for demographic parameters

\begin{tabular}{|l|c|c|}
\hline \multicolumn{1}{|c|}{ Parameters } & Mean \pm SD & Range \\
\hline Age (in years) & $40.99 \pm 10.99$ & $21-60$ \\
\hline Gender & No. & $(\%)$ \\
\hline Male & 208 & 46.2 \\
\hline Female & 242 & 53.8 \\
\hline
\end{tabular}

Table 1 provides the descriptive statistics for demographic parameters. The mean age of the study groups was $40.99 \pm 10.99$ years. Among the study groups $46.2 \%$ were males, $53.8 \%$ were females.

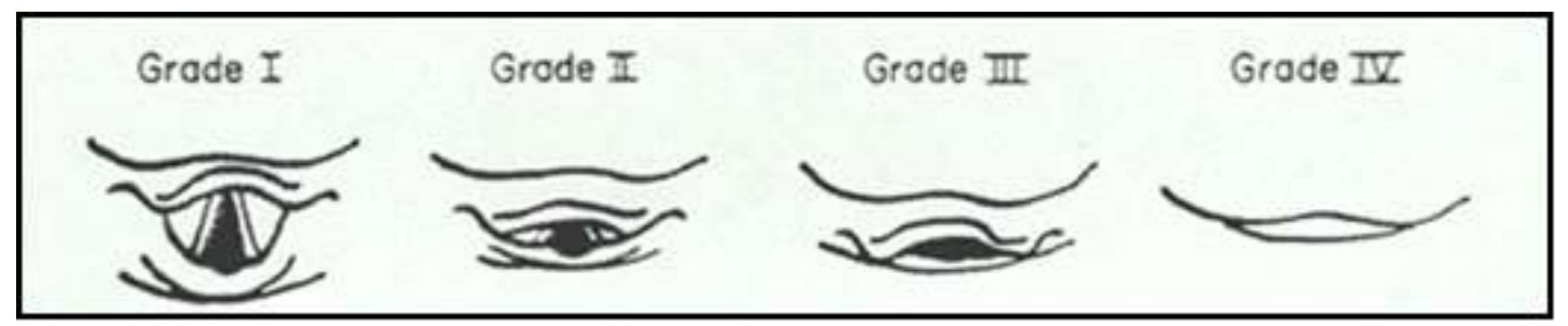

Fig. 4: Cormack-Lehane grading system 
Table 2: Cormack-Lehane grading comparison with demographic parameters

\begin{tabular}{|l|c|c|c|}
\hline \multicolumn{1}{|c|}{ Parameters } & $\begin{array}{c}\text { Easy } \\
(\mathbf{n = 4 2 0})\end{array}$ & $\begin{array}{c}\text { Difficult } \\
(\mathbf{n = 3 0 )}\end{array}$ & P-value* \\
\hline Age (in years) [mean \pm SD] & $39.96 \pm 10.49$ & $55.93 \pm 5.66$ & $<\mathbf{0 . 0 0 1}(\mathbf{S})$ \\
\hline Gender [No. (\%)] & & & \\
\hline Male & $190(45.2)$ & $18(60.0)$ & \multirow{2}{*}{$0.168(\mathrm{NS})$} \\
\hline Female & $230(54.8)$ & $12(40.0)$ & \\
\hline
\end{tabular}

*Obtaining using Chi Square test and independent t-test; S: Significant; NS: Not Significant

Table 2 provides the descriptive statistics according to $\mathrm{C}-\mathrm{L}$ grading. Based on Laryngoscopic grading method to assess difficulty in intubation there was significant difference in age with P-value $<0.05$. For gender distribution, there was insignificant difference with p-value of 0.168 by using chi square test.

Table 3: Comparison of various predictive tests with Cormack-Lehane grading and estimation of kappa coefficients

\begin{tabular}{|c|c|c|c|c|c|}
\hline \multirow{3}{*}{ Predictive tests } & \multirow{3}{*}{ Intubations } & \multicolumn{2}{|r|}{ C-L grading } & \multirow{3}{*}{ Kappa coefficient } & \multirow{3}{*}{ P-value* } \\
\hline & & Difficult $(n=30)$ & $\operatorname{Easy}(n=420)$ & & \\
\hline & & No. $(\%)$ & No. $(\%)$ & & \\
\hline \multirow[t]{2}{*}{ MMT } & Difficult & $30(100.0)$ & $8(1.9)$ & \multirow{2}{*}{$0.87(0.79-0.96)$} & \multirow{2}{*}{$<0.001(\mathrm{~S})$} \\
\hline & Easy & 0 & $412(98.1)$ & & \\
\hline \multirow[t]{2}{*}{ ULBT } & Difficult & $7(23.3)$ & 0 & \multirow{2}{*}{$0.36(0.17-0.56)$} & \multirow{2}{*}{$<0.001(\mathrm{~S})$} \\
\hline & Easy & $23(76.7)$ & $420(100.0)$ & & \\
\hline \multirow[t]{2}{*}{ TMD } & Difficult & $17(56.7)$ & $2(0.5)$ & \multirow{2}{*}{$0.68(0.52-0.83)$} & \multirow{2}{*}{$<0.001(\mathrm{~S})$} \\
\hline & Easy & $13(43.3)$ & $418(99.5)$ & & \\
\hline \multirow[t]{2}{*}{ RHTMD } & Difficult & $26(86.7)$ & $4(1.0)$ & \multirow{2}{*}{$0.86(0.76-0.95)$} & \multirow{2}{*}{$<0.001(\mathrm{~S})$} \\
\hline & Easy & $4(13.3)$ & $416(99.0)$ & & \\
\hline
\end{tabular}

S: Significant

Table 3 provides the comparison of different tests with C-L grading and estimation of kappa coefficients. In this study comparing C-L grade with other test such as MMT, ULBT, TMD, and RHTMD in assessing the difficulty in intubation, it was observed that there was statistically significant association with p-value $<0.05$. Kappa coefficient was highest for MMT and RHTMD with C-L grading as compare to other tests.

Table 4: Sensitivity, specificity, PPV, NPV of various predictive test

\begin{tabular}{|l|c|c|c|c|c|}
\hline Laryngoscopic view & Sensitivity (\%) & Specificity (\%) & PPV (\%) & NPV (\%) & Accuracy \\
\hline MMT & $100(88-100)$ & $98.10(96-99)$ & $79(63-90)$ & $100(99-100)$ & 98.2 \\
\hline ULBT & $23.33(10-42)$ & $100(99-100)$ & $100(59-100)$ & $95(92-97)$ & 94.9 \\
\hline TMD & $56.67(37-75)$ & $99.52(98-100)$ & $89(67-99)$ & $97(95-98)$ & 96.7 \\
\hline RHTMD & $86.67(69-96)$ & $99.05(98-100)$ & $87(69-96)$ & $99(98-100)$ & 98.2 \\
\hline
\end{tabular}

Table 4 provides the sensitivity, specificity, positive predictive value, negative predictive value and accuracy of different predictive tests MMT, ULBT, TMD and RHTMD with Laryngoscopic view in predicting difficulty in intubation. MMT had highest sensitivity (100\%), ULBT had highest specificity (100\%) and positive predicted value (100\%), and MMT had highest negative value (100\%). MMT and RHTMD had highest diagnostic accuracy followed by other tests.

Table 5: Sensitivity, specificity, PPV and NPV of airway predictive test in combinations

\begin{tabular}{|l|c|c|c|c|}
\hline \multicolumn{1}{|c|}{ Airway Tests } & Sensitivity (\%) & Specificity (\%) & PPV (\%) & NPV (\%) \\
\hline ULBT+MMT & 100 & 98 & 79 & 100 \\
\hline ULBT+TMD & 67 & 100 & 100 & 97.6 \\
\hline ULBT+RHTMD & $\mathbf{9 0}$ & $\mathbf{9 9}$ & $\mathbf{8 7 . 1}$ & $\mathbf{9 9 . 2}$ \\
\hline ULBT+MMT+TMD & 100 & 98 & 79 & 100 \\
\hline ULBT+MMT+RHTMD & 100 & 97 & 71.5 & 100 \\
\hline
\end{tabular}


Table 5 provides the sensitivity, specificity, positive predictive value, negative predictive value of airway predictive tests in combinations. Combination of ULBT and MMT test, combination of ULBT, MMT and TMD as well as combination of ULBT, MMT and RHTMD had $100 \%$ sensitivity. The combination ULBT and RHTMD had a sensitivity of $90 \%$, while ULBT and TMD had $67 \%$.

As regards specificity, ULBT and TMD had the highest specificity of $100 \%$, followed by ULBT and RHTMD with 99\%, ULBT and MMT as well as ULBT, MMT and TMD with $98 \%$ each, and followed by ULBT, MMT and RHTMD with $97 \%$.

The positive predictive value of ULBT and TMD was $100 \%$, followed by ULBT, RHTMD with $87.1 \%$, ULBT and MMT as well as ULBT, MMT and TMD with 79\%.

The negative predictive value for ULBT and MMT, ULBT, MMT and TMD as well as ULBT, MMTT and RHTMD was $100 \%$, followed by ULBT and RHTMD with 99.2\%, and ULBT and TMD with $97.6 \%$.

\section{Statistical Method}

Sensitivity and Specificity for combination of tests

$$
\begin{aligned}
& \text { Sensitivity } \text { of combination of tests }=1-\prod_{i=1}^{n}\left(1-\text { sensitivity }_{\text {Test }_{S}}\right) \\
& \text { Specificity of combination of tests }=1-\prod_{i=2}^{n}\left(1-\text { specificity }_{\text {Tes, }_{1}}\right)
\end{aligned}
$$

where $i=1,2, \ldots n$ indicate the number of tests used.

\section{Discussion}

Unexpected difficult intubations are probably the result of lack of accurate predictive tests for difficult intubation and inadequate preoperative examinations of the airway.

Ideally, any preoperative assessment of difficult tracheal intubation should have high sensitivity and specificity to result in minimal false positive or negative values. Sensitivity and specificity are dependent on each other, an increase in one of them usually results in decrease of other.

The current study was undertaken to study the predictive values as well as to elucidate the role of MMT, ULBT, TMD and RHTMD as simple bedside airway predictive tests in prediction of preoperative difficult airway assessment.

We hypothesised that there will be a significant direct relationship between the MMT, ULBT, TMD RHTMD and Cormack and Lehane scale. To test the validity of this hypothesis, we conducted this study in patients undergoing general anaesthesia. We used the Cormack-Lehane system as the gold standard for testing the validity of MMT, ULBT, TMD and RHTMD so, in this study 450 subjects were classified into 'Easy' and 'Difficult' groups based on degree of difficulty in intubation. There were 420 cases of easy intubation, while 30 cases of difficult intubation. The mean age of patients in the easy group was $39.96 \pm 10.49$ years, while that of the difficult group was $55.93 \pm 5.66$ years. Difference in the means was statistically significant with Pvalue $<0.001$.
Similarly in the study conducted by Prakash et al. ${ }^{3}$ it was observed an association between difficult laryngoscopy and old age. For the age-related increase in difficult laryngoscopy they have mentioned osteoarthritic changes and poor dentition is the major responsible factor.

The gender distribution in two groups was insignificantly different with p-value of 0.168 as per Chisquare test. Savva et al. ${ }^{4}$ also did not report any difference in age, sex, weight or height between easy and difficult laryngoscopy groups.

\section{Upper LIP Bite Test}

ULBT has the potential to evaluate both jaw movement and buck teeth simultaneously, providing additional support for its use as an airway assessment test.

ULBT has many obvious advantages. Firstly, it is a simple bedside method that involves the assessment of jaw subluxation and presence of buck teeth. Secondly, the three classes are clearly demarcated and delineated, making interobserver variations highly unlikely and its use is not dependent on skill or experience level.

In the present study ULBT had the least sensitivity of $23.33 \%$, specificity of $100 \%$, PPV of $100 \%$, NPV of $95 \%$ and accuracy of $94.9 \%$. This is in contrast to the results obtained by Khan et al, ${ }^{5}$ Azmat Ali et al, ${ }^{6}$ Ali et $\mathrm{al}^{7}$ and Karci et $\mathrm{al}^{8}$ wherein they found a sensitivity of $76.5 \%$, $91.5 \%, 87.5 \%$, and $13 \%$ respectively.

Khan et $\mathrm{al}^{5}$ comdpare ULBT with MMT and concluded that the upper lip bite test with sensitivity $76.5 \%$, specificity 88.7\%, PPV $28.9 \%$ and NPV $98.4 \%$ is an acceptable option for predicting difficult intubation as a simple, single test.

Azmat Ali et $\mathrm{al}^{6}$ calculated accuracy, sensitivity, specificity, positive predictive value and negative predictive value of ULBT which were $95.5 \%, 91.5 \%, 96 \%, 72.8 \%$ and 98.9\% respectively. They found that ULBT is a highly accurate, sensitive and specific test for predicting difficult intubations.

Ali et $\mathrm{al}^{7}$ obtained accuracy $91.9 \%$, sensitivity $87.5 \%$, specificity $92.9 \%$, PPV $71.6 \%$ and NPV $97.3 \%$ of upper lip bite test. They mentioned the probable reasons for high sensitivity $(87.5 \%)$ was lack of inter-observer variance as well as ethnic difference.

Our study with sensitivity of ULBT $23.33 \%$, is in concordance with the study done by Eberhart et $\mathrm{al}^{9}$, wherein they found sensitivity of $26.2 \%$. They found major reason for this failure to be a more frequent incidence of difficult laryngoscopy in study patients. They hypothesized that this was because of the variability of clinical experience in the anaesthesiologists performing the endotracheal intubation.

The specificity of ULBT in our study was $100 \%$, which correlates with the studies done by Khan et $\mathrm{al}^{5}(88.7 \%)$, Eberhart et $\mathrm{al}^{9}(92.5 \%)$, Hester et $\mathrm{al}^{10}(97 \%)$.

The lower sensitivity $23.3 \%$ of ULBT in our study can be explained due to low incidence of ULBT Class III in our study i.e. 7 out of 450 patients. (Table 3 )

We found that repeated demonstrations were required for the patients to perform ULBT and a few still failed to understand the procedure inspite of our efforts. Also in 
some, there was a reflex movement of upper lip in the reverse direction over the upper teeth which may have altered the point of meeting of vermilion line with lower incisors.

\section{Modified Mallampati Test (MMT)}

Modified Mallampati Test (MMT) assessment gives significant correlation between the ability to visualize pharyngeal structures and ease of laryngoscopy and intubation. Because, as the degree to which oropharyngeal structures could be visualized upon examination should correlate with structures that could be on laryngoscopy. Disadvantage is that, MMT does not consider the patient's dentition or variation in the degree of mandibular range of motion and the patients with small mouth opening or altered level of consciousness could be misclassified, hence its validity of assessment is influenced by the experience and the skill of the anesthetist. The specificity of Modified Mallampati test (MMT) was $100 \%$ in our study. This is in contrary to the results obtained by Khan et al, ${ }^{5}$ Eberhart et $\mathrm{al}^{9}$ and Hester et $\mathrm{al}^{10}$ wherein they reported specificity of MMT as $66.8 \%, 61.0 \%$, and $75 \%$ respectively.

Hester et al $^{10}$ obtained sensitivity $11 \%$, specificity $75 \%$, PPV 9\%, NPV 79\%. They suggest that MMT is a subjective instrument in predicting difficult airway, with inter-observer variation significantly altering the results.

This discrepancy may be explained by the fact that in our study both the preoperative evaluation of airway predictors and intubation were done by different persons. So the chances of inter-observer bias as reported by many authors may be an issue here. ${ }^{5}$

\section{Thyromental Distance and Ratio of Height to Thyromental Distance (RHTMD)}

The TMD was measured with the head fully extended and the mouth closed. For the thyromental distance (TMD) varying "critical distances", ranging from 5.5 to $7 \mathrm{~cm}$ are used to predict a difficult laryngoscopy. ${ }^{2}$ This makes comparison of results difficult and questions the predictive value of this test.

Based on the theorized relationship between patient's height and TMD, the ratio of height and TMD (RHTMD) might improve prediction. Schmitt et al. ${ }^{2}$ showed that the ratio of height to TMD (RHTMD) had a better predictive value than the TMD.

In the present study sensitivity, specificity for thyromental distance (TMD) was $56.67 \%$ and $99.52 \%$ respectively. In a study Salimi et $\mathrm{al}^{11}$ reported a sensitivity of $55 \%$ and specificity of $88 \%$, Khan et al ${ }^{12}$ reported sensitivity of $73 \%$ and specificity of $82.2 \%$ for TMD.

In a study Salimi et al $^{11}$ reported a sensitivity of $55 \%$ and specificity of $88 \%$. The low sensitivity obtained in their study might be due to anthropometric peculiarities in the study population.

So, variation in reported sensitivity in our study as well as various other studies may be because of anthropometric peculiarities. ${ }^{11}$
RHTMD had the sensitivity of $86.67 \%$ and specificity of $99.05 \%$. Schmitt et al. ${ }^{2}$ introduced RHTMD and found that it has good predictive value for predicting difficult laryngoscopy than TMD as it allows for individual's body proportions which are not allowed in TMD. Schmitt et al. ${ }^{2}$ got sensitivity $81 \%$ and specificity $91 \%$ for RHTMD which is quite similar with our study findings i.e., sensitivity of $86.67 \%$ and specificity of $99.05 \%$.

These findings are not consistent with those reported by Krobbuaban et al. ${ }^{13}$ (sensitivity, 77\%; specificity, 66\%), Shah et al. ${ }^{14}$ (sensitivity, $71.6 \%$; specificity, 92\%), Safavi et al. ${ }^{15}$ (sensitivity, $75.6 \%$; specificity, 58.5\%). Although the different statistical values in our study, like, sensitivity of $86.67 \%$ and specificity of $99.05 \%$ for RHTMD varied from other studies, the conclusion of RHTMD test better than TMD test was comparable.

\section{Kappa Coefficient}

Kappa is the most commonly reported measure in the medical literature for assessing interobserver agreement. The values of kappa range from -1 to +1 , with -1 indicating perfect disagreement and +1 indicating perfect agreement between the raters.

In this study, $\mathrm{C}-\mathrm{L}$ grading was considered as standard and all the other test results were compared with the standard. The comparison of ULBT with C-L grading resulted into a kappa coefficient of 0.36 with $\mathrm{P}$-value < 0.001 , indicating fair agreement. TMD test had the kappa value of 0.68 with $\mathrm{P}$-value $<0.001$, implying substantial agreement between the two tests. The kappa coefficient for MMT test was 0.87 with P-value $<0.001$. In other words, the agreement between MMT test grading and C-L grading was almost perfect. RHTMD had a kappa coefficient of 0.86 with $\mathrm{P}$-value $<0.001$, it also indicates almost perfect agreement with $\mathrm{C}-\mathrm{L}$ grading. (Table 4)

Preoperative airway assessment tests are screening tests, and therefore should be highly sensitive to predict the maximum number of patients of difficult laryngoscopy correctly and highly specific to predict easy laryngoscopy correctly. Test should also have a high PPV with few falsenegative predictions. NPV is the probability that patients with a negative screening test truly do not have difficult laryngoscopy.

MMT (Mallampati test) have sensitivity 100\%, specificity $98.10 \%$, PPV of $79 \%$, NPV of $100 \%$ and accuracy $98.2 \%$. It suggests that MMT can predict difficult and easy laryngoscopy correctly that were truly difficult and easy because of sensitivity $100 \%$ \& specificity $98.10 \%$ respectively. PPV 79\% shows that MMT predicting less number of intubations to be difficult. With NPV 100\%, MMT successfully predicted all intubations to be easy.

ULBT (Upper lip bite test) have sensitivity $23.33 \%$, specificity $100 \%$, PPV of $100 \%$, NPV of $95 \%$ and accuracy $94.9 \%$. With sensitivity of $23.3 \%$, ULBT has predicted less number of patients with difficult laryngoscopy correctly that were truly difficult. Specificity of $100 \%$ indicates that ULBT predicted all easy laryngoscopies correctly which 
were truly easy. PPV of $100 \%$ \& NPV of $95 \%$ indicates correct prediction of difficult and easy intubation.

TMD (thyromental distance) have sensitivity 56.7\%, specificity $99.5 \%$, PPV of $89 \%$, NPV of $97 \%$ and accuracy $96.7 \%$. Sensitivity of $56.7 \%$ indicates low prediction of difficult laryngoscopy that were truly difficult. This may be because of anthropometric peculiarities. Specificity of 99.5\% indicates prediction of almost all easy intubations that were truly easy. PPV of $89 \%$ indicates good percentages for correct prediction of difficult laryngoscopy.

RH MD (ratio of height to thyromental distance) have sensitivity $86.7 \%$ which indicates good percentage of intubation predicted difficult which were truly be difficult. With specificity of $99.05 \%$ this test had predicted nearly all easy intubation which were truly easy. PPV of $87 \%$, NPV of 99\% indicates good percentages for correct prediction of easy intubation. Accuracy of RHTMD is $98.2 \%$.

To summarise, our study shows MMT had highest sensitivity (100\%), ULBT had highest specificity (100\%) and positive predictive value (100\%), and MMT had highest negative predictive value (100\%). MMT and RHTMD with accuracy of $98.2 \%$ had highest diagnostic accuracy followed by other tests. (Table 6 )

So, safe outcome of anaesthesia continues to be an important goal for every anaesthesiologist. Unfortunately, there is still no test that can predict $100 \%$ of difficult laryngoscopies. Therefore we studied combination of various assessment methods in predicting the ease of intubation for improving the sensitivity rates.

We made 5 combinations of MMT, ULBT, TMD \& RHTMD, as follows-

ULBT + MMT.

ULBT + TMD.

$\mathrm{ULBT}+$ RHTMD.

$\mathrm{ULBT}+\mathrm{MMT}+\mathrm{TMD}$

$\mathrm{ULBT}+\mathrm{MMT}+\mathrm{RHTMD}$.

All these tests have their own statistical and predicitive values (sensitivity, specificity, positive predictive value PPV, negative predictive value NPV) which are different from each other. Like in our study, MMT had highest sensitivity (100\%), ULBT had highest specificity (100\%) and positive predictive value (100\%), and MMT had highest negative predictive value (100\%). (Table 5)

Combination of these tests was difficult due to above mentioned factors. So, to overcome this we considered these tests almost equivalent to obtain standardised statistical values. Hence, comparison of combinations of different airway predictive tests was possible.

ULBT + TMD combination has sensitivity 67\%, specificity $100 \%$, positive predictive value $100 \%$, negative predictive value $97.6 \%$. This combination provides almost perfect specificity (100\%), positive predictive value $(100 \%)$, negative predictive value $(97.6 \%)$ but with lowest sensitivity (67\%) among the combinations we made. So, it has not correctly predicted difficult laryngoscopies as a proportion of all laryngoscopies that were truly difficult. Hence, this combination stands last in order of choice.
$\mathrm{ULBT}+\mathrm{MMT}+\mathrm{RHTMD}$ combination has sensitivity $100 \%$, specificity $97 \%$, positive predictive value $71.5 \%$ and negative predictive value $100 \%$. This combination have lowest positive predictive value of $71.5 \%$ among other combinations. So, it has not given the percentage of correctly predicted difficult laryngoscopies as a proportion of all predicted difficult laryngoscopies. This combination gives better results than ULBT + TMD. So ULBT + MMT + RHTMD is better combination than ULBT + TMD.

$\mathrm{ULBT}+\mathrm{MMT} \& \mathrm{ULBT}+\mathrm{MMT}+\mathrm{TMD}$, both of these combinations have sensitivity $100 \%$, specificity $98 \%$, positive predictive value $79 \%$ and negative predictive value $100 \%$. These two combinations are near to ideal airway prediction model but with positive predictive value of $79 \%$ it has not correctly predicted difficult laryngoscopies that were truly difficult. This combination is better than both the combinations discussed above.

ULBT + RHTMD combination has sensitivity $90 \%$, specificity $99 \%$, positive predictive value $87.1 \%$ and negative predictive value $99.2 \%$. This combination we think is ideal airway prediction model because though it has comparatively less sensitivity $90 \%$, with positive predictive value $87.1 \%$, specificity $99 \%$ and negative predictive value $99.2 \%$, it predicts all difficult and easy intubations correctly.

Hence, ULBT + RHTMD with sensitivity 90\%, specificity $99 \%$, PPV $87.1 \%$ \& NPV $99.2 \%$; is the best combination among the combinations we made.

Such a combination is preferable because anatomic predictors of difficult intubation carry a low-sensitivity rate when used alone, whereas a multivariate composite risk index may achieve better results than single, independent criteria. ${ }^{10}$ We suggest that further more studies needs to be carried out to prove the efficacy of such combinations of airway assessment tests for the prediction of difficult airway and intubation.

\section{Conclusion}

We conclude that no single airway predictor is sufficient for predicting difficult intubation. A different combination of two OR more airway predictor have to be taken into consideration to arrive at near ideal airway prediction model.

So, in our study we found ULB + RHTMD is the best combination.

Conflict of Interest: None.

\section{References}

1. Merah NA, Wong DT, Foulkes-Crabbe DJ. Modified Mallampatti test, thyromental Distance and inter-incisor gap are the best predictors of difficult laryngoscopy in West Africans. Can J Anesth 2005; 52;291-6.

2. Schmitt HJ, Kirmse M, Radespiel-Troger M Ratio of patient's height to thyromental distance Improves prediction of difficult laryngoscopy. Anaesth Intensive Care 2002;30;763-5.

3. Prakash S, Kumar A, Bhandari S, Mullick P, Singh R, Gogia AR. Difficult laryngoscopy and intubation in the Indian population: An assessment of anatomical and clinical risk factors. Indian J Anaesth 2013;57(6):569. 
4. Savva D. Prediction of difficult tracheal intubation. Br J Anaesth1994;73;149-53.

5. Khan ZH, Kashif A, Ebrahimkhani E.A comparison of upper lip bite test (a simple new technique) with modified Mallampati classification in predicting difficulty in endotracheal intubation: A perspective blinded study. Anesth Analg 2003;96;595-99.

6. Azmat A S, Khalid R, Mujahid I. Can difficult intubation be accurately predicted using upper lip bite test? J Postgrad Med Inst 2014;28:282-7.

7. Ali MA, Qamar-ul-Hoda M, Samad K. Comparison of upper lip bite test with Mallampati test in the prediction of difficult intubation at a tertiary care hospital of Pakistan. J Pak Med Assoc 2012;62:1012-5.

8. Karci A, Karagöz S, Girgin P, Bozdogan DG. Comparison of Modified Mallampati classification, upper lip bite test and neck circumference in prediction of difficult intubation. Eur $J$ Anaesthesiol 2011;28:236.

9. Eberhart LH, Arndt C, Cierpka T, Schwanekamp J, Wulf H, Putzke C. The reliability and validity of the upper lip bite test compared with the Mallampati classification to predict difficult laryngoscopy an external prospective evaluation. Anesth Analg 2005;101:284-9.

10. Hester CE, Dietrich SA, White SW, Secrest JA, Lindgren KR, Smith T. A comparison of preoperative airway assessment techniques the modified Mallampati and the upper lip bite test. AANA J 2007;75:177-82.

11. Salimi A, Farzanegan B, Rastegarpour A, Kolahi AA. Comparison of the upper lip bite test with measurement of thyromental distance for prediction of difficult intubations. Acta Anaesthesiol Taiwan 2008;46:61-5.

12. Khan ZH, Mohammadi M, Rasouli MR, Frrokhnia F, Khan RH. The diagnostic value of the upper lip bite test combined with sternomental distance, thyromental distance, and interincisor distance for prediction of easy laryngoscopy and intubation: A prospective study. Anesth Analg 2009;109:8224.

13. Krobbuaban B, Diregpoke S, Kumkeaw S, Tanomsat M. The predictive value of the height and thyromental distance: four predictive tests for difficult laryngoscopy. Anesth Analg 2005;101:1542-5.

14. Shah PJ, Dubey KP, Yadav JP. Predictive value of upper lip bite test and ratio of height to thyromental distance compared to other multivariate airway assessment tests for difficult laryngoscopy in apparently normal patients. J Anaesthesiol Clin Pharmacol 2013;29:191-5.

15. Safavi M, Honarmand A, Zare N. A comparison of the ratio of patient's height to thyromental distance with the modified Mallampati and the upper lip bite test in predicting difficult laryngoscopy. Saudi J Anaesth 2011;5:258-63.

How to cite this article: Bhure A, Wasnik A, Deshmukh PP, Tiwari Y. Comparative study of airway assessment tests to predict difficult laryngoscopy \& intubation. Indian $J$ Clin Anaesth 2019;6(2):172-9. 